\title{
Einige Eigenschaften einer gewissen Gattung von Curven vierter 0rdnung.
}

\author{
Von J. Lürote in KarLsRuHe.
}

Herr Clebsch hat in seinem Aufsatze über die Theorie der Curven vierter Ordnung (Crelle's Journal Bd. 59) gezeigt, dass es trotz der in genügender Anzahl vorhandenen Constanten im Allgemeinen nicht möglich sei, die Gleichung einer Curve vierter Ordnung darzustellen als eine Summe von fünf vierten Potenzen; dass vielmehr diejenigen Curven, welche diese Eigenschaft besitzen, sich auszeichnen durch das Verschwinden einer Invariante. Herr Cle.bs ch hat im citirten Aufsatze einige Eigenschaften dieser Curven abgeleitet; im Folgenden sollen einige andere angegeben und besonders der Beweis geführt werden, dass das Verschwinden jener Invariante auch eine hinreichende Bedingung ist zur Darstellung der Curve als Summe von fünf Biquadraten.

\section{§. 1 .}

\section{Darstellung der Gleichung als Summe von fünf Quadraten.} Form

Schreiben wir die Gleichung einer Curre vierter Ordnung in der

$$
u=\sum_{i, k, l, m} u_{i k l m} x_{i} x_{k} x_{l} x_{m}
$$

wo sich die Summe erstreckt über die Werthe 1,2,3 der Indices, so sind die Curven, welche wir hier betrachten wollen, charakterisirt durch die Bedingung, dass die Determinante

$$
A=\left|\begin{array}{llllll}
u_{1111} & u_{11 \text { 12 }} & u_{1122} & u_{1113} & u_{1123} & u_{1133} \\
u_{1211} & u_{1212} & u_{1222} & u_{1213} & u_{1223} & u_{1233} \\
u_{2211} & u_{2212} & u_{2222} & u_{2213} & u_{2223} & u_{2233} \\
u_{1311} & u_{1312} & u_{1322} & u_{1313} & u_{1323} & u_{1333} \\
u_{2311} & u_{2312} & u_{2322} & u_{2313} & u_{2323} & u_{2333} \\
u_{3311} & u_{3312} & u_{3322} & u_{3313} & u_{3323} & u_{3333}
\end{array}\right|,
$$


welche eine Invariante ist, verschwindet. Bezeichnen wir die zum Elemente $u_{i k, l m}$ gehörige Unterdeterminante von $A$ mit $A_{i k, l m}$, so ist $A_{i k, l m}=A_{l m, i k}$, and diese Grössen haben, woil $A=0$, die Eigenschaft, dass sechs Grössen $p_{i k}$ existiren, welche die Gleichungen

erfüllen.

$$
A_{i k, l m}=p_{i k} p_{i m} \quad i, k, l, m=1,2,3
$$

Diese sechs Grössen $p_{i k}$ kann man betrachten als Coëfficienten der Gleichung einer Curve zweiter Classe $K$, welche dann eine zugehörige Form sein wird.

Wir fassen nun die Polare eines Punktenpaares $x y$ in's Auge, d. h. die erste Polare des einen dieser Punkte in Bezug auf die erste Polare des zweiten; deren Gleichung ist

$$
\Sigma \eta_{i} \eta_{k} x_{l} y_{s n} u_{i k l m}=\Sigma a_{i k} \eta_{i} \eta_{k}=0 \text {. }
$$

Diese Polare ist ein Kegelschnitt, dessen Coëfficienten sind:

$$
a_{i k}=\sum_{i, m} x_{l} y_{m} u_{i k l m}
$$

Múltipliciren wir mit $p_{i k}$ und summiren nach $i$ and $k$, so erhalten wir, wegen der Definition der $p_{i k}$, die Gleichung:

$$
\sum_{i k} a_{i k} p_{i k}=0 \text {. }
$$

Wenn umgekehrt die Coëfficienten $a_{i k}$ eines beliebigen Kegelschnittes diese Gleichung erfüllen, so kann er als zweite Polare aufgefasst werden. Denn aus den obigen Gleichungen, die dann nur fünf unabhängige darstellen, folgen fünf der Grössen:

$$
2 x_{1} y_{1}, x_{1} y_{2}+x_{2} y_{1}, 2 x_{2} y_{2}, x_{1} y_{3}+x_{3} y_{1}, x_{2} y_{3}+x_{3} y_{2}, 2 x_{3} y_{3}
$$

als lineare Functionen der sechsten. Die Determinante dritten Grades dieser sechs Grössen, welche bekanntlich verschwindet, liefert dann eine Gleichung' dritten Grades für diese sechste Grösse, wodarch diese bestimmt ist. Wenn man sich erinnert an die von Herrn Hesse'gegebene geometrische Deutung der Gleichung (3), so erhält man den Satz: Wenn ein gegebener Kegelschnitt Polare eines Punktenpares sein soll, so muss ein Polardrejeck von $K$ ihm eingeschrieben oder eines seiner Polardreiecke $K$ umgeschrieben werden können. Es gibt dann 3 Punktenparar, als deren Polare der Kegelschnitt betrachtet werden kann.

Wix bezeichnen jetzt die Coordinaten von sechs beliebigen Punktenpaaren mit $x^{k} y^{3}(h=1,2,3,4,5,6)$ und multipliciren die Determinante $A$ mit einer andern, in der eine Zeile ist:

$x_{1}^{h} y_{1}^{h}, x_{1}^{h} y_{2}^{h}+x_{2}^{h} y_{1}^{h}, x_{2}^{h} y_{2}^{h}, x_{1}^{h} y_{3}^{h}+x_{3}^{h} y_{1}^{h}, x_{2}^{h} y_{3}^{h}+x_{3}^{h} y_{2}^{h}, x_{3}^{h} y_{3}^{h}$. Wir erhalten auf diese Weise eine neue Determinante, in der die Elemente der $h^{\text {ten }}$ Zeile entstehen ans: 


$$
\sum_{l, m} x_{l}^{h} y_{m}^{h} u_{z k l m}
$$

indem man für $i, k$ die Werthe 1,2,3 setzt. Da diese Determinante aber mit $A$ gleichzeitig verschwindet, so kann man sechs Coëfficienten $p_{h}$ so bestimmen, dass

$$
\sum_{h} p_{h} \underset{l_{2} m}{\Sigma} u_{i k \ell m} x_{l}^{h} y_{m}^{h}=0 \quad i, k=1,2,3 .
$$

Multiplicirt man diese Gleichung mit $\eta_{i} \eta_{k}$ and summirt nach $i$ und $\gamma_{\text {, }}$ so entsteht die didentische Gleichung:

$$
\sum_{h} p_{h} \sum_{i l / m} \eta_{i} \eta_{k} x_{l}^{h} y_{m}^{h} u_{i k l m}=0 \text {, }
$$

wo die $p_{h}$ Functionen sind der Coordinaten der sechs Punktenpaare. Diese Gleichung lehrt, dass die Gleichung der Polare eines beliebigen Punktenpares sich linear ausdrücken lässt durch die Gleichungen der Polaren ron fünf anderen beliebigen Punktenpa aren. Und wenn umgekehrt diese Eigenschaft stattfindet, so zeigt die vorhergehende Ableitung, dass auch die Invariante $A$ verschwindet, den Fall ausgenommen, dass die Determinante, mit der oben multiplicirt wurde, selbst verschwindet. Dies tritt aber ein, wenn die sechs Punktenpaare harmonische Polenpaare eines und desselben Kegelschnittes sind; und in diesem speciellen Falle gilt der vorige Satz für alle Curven vierter Ordnung.

Die Coëfficienten $p_{h}$ bestimmen sich am einfachsten, wenn man die fünf Punktenpaare passend wählt und zwar so, đass jedes Paar ein harmonisches Polenpaar ist der Polaren der anderen Punktenpaare. Dass sich solche fünf Polenpaare stets angeben lassen, zeigt sich leicht mit Hülfe des Satzes: Wenn zwei Punkte $x, y$ harmonische Pole sind der Polare zweier anderen Punkte $\xi, \eta$, so sind umgekehrt $\xi, \eta$ harmonisehe Pole der Polare von $x, y$, den die doppelte Interpretation der Gleichung

$$
\sum \xi_{i} \eta_{k} x_{l} y_{m} u_{i k l m}=0
$$

ergibt. Denn man gehe von einem Punktenpaare 1 aus und nehme ein harmonisches Polenpaar seiner Polare zum Punktenpare 2. Den einen Punkt des Paares 3 kann man noch beliebig wählen, der zweite ist aber dann bestimmt als Schnitt der Polaren des ersten Punktes in Bezug auf die Polaren von 1 und 2. Wenn man nun zu den drei Kegelschnitten 1,2,3 die Ja co bi'sche Curve construirt, so kann man auf dieser eine unendliche Anzahl von Punktenpaaren angeben, welche harmonische Pole sind der drei Kegelschnitte 1,2,3. Eines dieser wählen wir zum Punktenpaare 4. Das fünfte Paar, welches harmonisch sein muss zu den 4 jetzt construirten Kegelschnitten, ist dreidentig bestimmt, wie ein Satz aus der Educational Times lehrt (ef. Crelle's Journal Bd. 68, p. 55). Der vorhin angeführte Satz zeigt 
jetzt, dass die fünf Punktenpare die Bedingung erfüllen, dass jedes harmonisch ist zu den Polaren der übrigen. Wir nehmen diese fünf Punktenpaare zn den Paaren $x^{4}, y^{1} \ldots x^{5} y^{5}$, setzen $p_{b}=-1$, and $x y$ für $x^{6} y^{6}$. Dann bestehen die Gleichungen

$$
\Sigma x_{i}^{h} y_{k}^{h} x_{l}^{h^{r}} y_{m}^{h^{\prime}} u_{i k l m k}=0 \text {, }
$$

wenn $h^{\prime}$ nicht $=h$ ist. Setzen wir nun in Gleichang (4), die jetzt lautet:

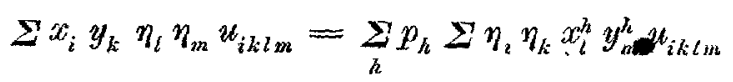

$x^{h}+\lambda y^{h}$ für $\eta$ und vergleichen beiderseits die Coëfficienten von $\lambda$, so erhalten wir, mit Rücksicht auf (5), die Gleichung:

$$
\Sigma x_{i}^{h} y_{k}^{h} x_{l} y_{m} u_{i k l m}=p_{h} \Sigma x_{i}^{h} y_{k}^{h} x_{l}^{h} y_{m}^{h} u_{i k l m} \text {. }
$$

Bezeichnen wir jetzat der Kürze wegen

so wird:

$$
\begin{array}{lll}
\Sigma x_{i}^{h} y_{k}^{h} \eta_{l} \eta_{\mu 2} u_{i k / m} & \text { mit } U_{h}(\eta \eta), \\
\sum x_{i}^{h} y_{k}^{h} x_{l} y_{m} u_{i k l m} & \text { mit } U_{h}(x y),
\end{array}
$$

$$
\sum \eta_{i} \eta_{k} x_{l} y_{u z} u_{i k l m}=\sum \frac{U_{h}(x, y)}{U_{h}\left(x^{h} y^{h}\right)} \cdot U_{h}(\eta \eta)
$$

Hiermit ist zugleich eine Bedingung gegeben für die Wahl der Punktenpaare. Da nämlich keiner der Nenner verschwinden soll, so darf keines der Paare so gewählt sein; dass der eine seiner Punlte auf der zweiten Polare des andern liegt.

Das sechste Paar $x, y$ ist noch ganz willkürlich. Man kann also auch den Punkt $x$ mit $y$ zusammenfallen lassen und hat dann, wenn man auch noch $\eta=x$ setzt, für den ganz willkürlichen Punkt $x$ die Gleichung:

$$
\sum x_{i} x_{k} x_{l} x_{m} u_{i k l m}=u=\sum_{h} \frac{U_{h}(x x)^{2}}{U_{h}\left(x^{h} y^{h^{2}}\right)} .
$$

Die Gleichung der Curve ist also ausgedrückt als Summe von fünf Quadraten. Wenn umgekehrt diese Darstellung möglich ist, so verschwindet, wie man sofort sieht, $A$. Addirt man nun zu der obigen Gileichung $\lambda U_{h}(x x)^{2}$, wo $\lambda$ ' beliebig, so erhält mañ die Gleichung des Curvenbüschels, dessen Curven die gegebene in den Punkten berühren, wo sie von dem Kegelschnitt $U_{h}(x x)=0$ getroffen wird. Da nun die so entstandene Gleichung sich noch als Summe von fünf Quadraten darstellt und $U_{h}(x x)$ als Gleichnng der zweiten Polare eines beliebigen Punktenpaares angesehen werden kann, so hat man den Satz: dass alle Curven vierter Ordnung, welehe die gegebene berühren in den Punkten, wo sie von der Polare eines beliebigen Punktenpares geschnitten wird, von der hier betrachteten Art sind. 
§. 2.

\section{Darstellung der Gleiehung der Curve als Summe von tïnf Biquadraten.}

Zunächst muss ich einige Sätze anführen über die Polaren von Punktempaaren, welche in zwei Linien oder eine Doppellinie zerfallen, und welehe Herr Clebsch a. o. a. 0 . bewiesen hat.

Wenn die Polare eines Punktenpaares zerfällt, so sind die beiden Linien, in welehe sie zerfällt, harmonische Polaren des Kegelschnittes $K$ und zu jedern solchen Paare von Polaren gehören drei Punktenpaare als Pole. Jeder Pol liegt auf der Determinante der ersten Polare (Polardeterminante) des andern. Jede Tangente von $K$ und nur eine solche kann als eine in eine Doppellinie ausgeartete Polare and zwar von drei Punktenpaaren betrachtet werden. Von den beiden Punkten eines solchen Paares ist jeder ein Eckpunkt der in drei Gerade zerfallenden Polardeterminante des anderen, und die ihm gegenüberliegende Seite dieses Dreiecks ist eben die Polare der beiden Punkte. Alle diese Punkte liegen auf einer Curve vierter Ordnung $S=0$ und jeder Punkt dieser Curve kann Punkt eines Paares sein. Die Seiten aller zerfallenden Polardeterminanten umhüllen also den Kegelschnitt $K$, und jede Tangente ist Seite von sechs Dreiecken, während die Ecken aller dieser Dreiecke aut $S$ liegen und jeder Punkt von $S$ Eckpunkt ron drei Dreiecken ist, deren Pole die Ecken seiner eigenen Polardeterminante sind.

Betrachten wir nun irgend eine Tangente $A_{1}$ des Kegelschnittes $K$. Diese muss nach dem Vorigen Seite von sechs zerfallenden Polardeterminanten sein, deren Ecken auf $S$ liegen müssen. 12 dieser Ecken sind also die Schnittpunkte von $A_{1}$ mit $S$ und nach dem Vorigen liegen in jedem dieser Schnittpunkte drei Ecken. Die Seiten der sechs Dreiecke gehen durch diese Ecken und berühren $K$, es sind also die Tangenten, welche man durch die Schnittpunkte ron $A_{1}$ mit $S$ an $K$ noch ziehen kann. Der Schnittpunkt je zweier ist ein Dreiechspunkt, der also auf $S$ liegen muss. Also bilden die vier Tangenten, welche mandurch die Schnittpunkteron $A_{1}$ und $S$ an $K$ legen kann, ein vollständiges Vierseit, dessen Ecken auf $S$ liegen und dessen Gegenecken die Pole der Tangente $A_{1}$ sind. Nennt man die vier mit Hülfe von $A_{1}$ construirten Tangenten $A_{2} A_{3} A_{4} A_{5}$, so erkennt man leicht, dass, wenn man von irgend einer derselben, $A_{2}$ z. B., ausgegangen wäre, man gerade die $A_{1} A_{3} A_{4} A_{5}$ gefunden hätte. Diese fünf Tangenten bilden also ein vollständiges Fünfseit, dessen 10 Ecken auf $S$ liegen. Die Polardeterminante irgend einer Ecke ist gebildet dureh diedreiseiten, welehe nicht dureh jene Eckegehen, und die Polare zweier 
Ecken, in welchen sich vier Seiten schneiden, bestebt aus der doppelt zu rechnenden fünften Seite. Die angegebene Construction zeigt ferner, dass das Fünfseit durch eine seiner Seiter oder eine seiner Ecken eindeutig bestimmt ist und dass man $S$ unendlich viele Fünfseite einsehreiben kann, welche $K$ umschrieben sind.

Man theile nun die 10 Eckpunkte des Fünfseits so in fünf Paare, dass jeder Seite des Fünfseits die Punkte eines Paares als Pole entsprechen. Bezeichnet man mit $x^{h} y^{h}$ die Pole $\operatorname{der}$ Seite $A_{k}$, so ist also

$$
\Sigma x_{i}^{h} y_{k}^{h} \eta_{l} \eta_{m} u_{i k l m}=A_{h}(\eta)^{2}
$$

das Quadrat der Gleichung dieser Seite. Es besteht dann die Gleichung (5)

$$
\Sigma x_{i}^{h} y_{k}^{h} x_{l}^{h^{\prime}} y_{m}^{h^{\prime}} u_{i k / m}=A_{h}\left(x^{h^{\prime}}\right) A_{h}\left(y^{h^{\prime}}\right)=0 \text {, }
$$

weil stets einer der Pole von $A_{k^{*}}$ auf der Seite $A_{k}$ liegt. Die fünt Punktenpaare köunen also an Stelle der im vorigen §. gebranchten treten und man erbält so die Gleichung:

$$
u=\sum_{h} \frac{A_{h}(x)^{*}}{A_{h}\left(x^{h}\right) A_{h}\left(y^{h}\right)} .
$$

Hiermit ist gezeigt, dass die Gleichnng unserer Curve sich darstellen lässt als Summe ron fünf Biquadraten und dass das Verschwinden der Invariante $A$ nothwendige und hinreichende Bedingung daza ist.

Durch Addition eines Gliedes $\varrho A_{h}(x)^{\ddagger}$ erkennt man, dass alle Curven vierter Ordnung, welche diese vierpunktig berïh. ren in den Pankten einer Tangente ron $K$, Curvendergleichen Art sind, wie die gegebere.

Die obige Darstellung verliert ibre Gültigkeit, wenn einer der Nenner verschwindet, $d, h$. wenn einer der Pole einer Seite in diese selbst fällt. Dies kann nur dann eintreten, wewn qwei Seiten eines Fünfseits

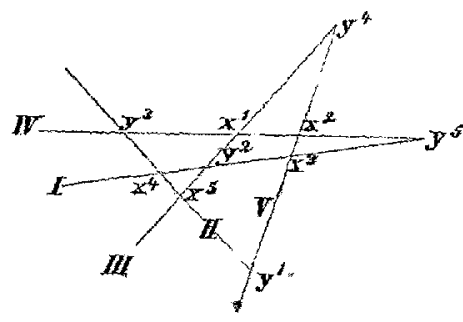
zusammenfallen. Fallen z. B. die beiden Seiten I und IV der Figur zusammen, so rücken die Punkte $y^{3} x^{1} x^{2}$ unendlich nahe resp. an die Punkte $x^{4} y^{2} x^{3}$, d. h. die Linien $11 ; I I T, V$ werden Tangenten von $S$. Da I und:IV zwei unendlich nahe Tangenten von $K$ sind, so muss ihr Schnittpunkt, der auf $S$ liegt, zugleich auf $K$ hegen.

Und amgekehrt ist unsehwer za erkennen, dass jedem Schnittpunkte von $K$ and $S$ ein Fünfseit entspricht, in welchem zwei Seiten zasam. menfallen, so dass deren Zaht acht beträgt. Jede dem Curven $S$ und $K$ gemeinsame Tangente liefert ebenfalls ein Fünfseit mit zusammenfallenden Seiten, das aber ausser ihr noeh zwei andere solche Tangenten 
enthält. Die Anzahl derselben wird also auch hier $=\frac{2.12}{3}=8$, wie oben. Diese Uebereinstimmung zeigt auch, dass $S$ keinen Doppelpunkt hat. Es gibt also acht Fünfseite, in welchen-zwei Seiten zusammenfallen. Diese Seite schneidet $S$ in vier Punkten, von welchen einer auf $K$ liegt. Die Tangenten, die mau in den drei anderen Schnittpunkten an $S$ legen kann, sind die drei anderen Seiten des Fünfseits, und ihre drei.Schnittpunkte liegen also auf $S$.

Betrachten wir non die erste Polare eines beliebigen Punktes $y$. Die Gleichung derselben ist:

$$
\Sigma y_{i} x_{k} x_{l} x_{m} u_{i k l m}=0
$$

Soll diese Polare einen Doppelpunkt haben in $x$, so muss

$$
\begin{aligned}
\sum_{i k} y_{i} x_{k} u_{i k l m} & =\alpha_{l} \beta_{m}+\alpha_{m} \beta_{i} \\
\Sigma \alpha_{i} x_{i} & =0 \\
\Sigma \beta_{i} x_{i} & =0
\end{aligned}
$$

sein. Die Tangenten des Doppelpunktes müssen also reciproke Polaren von $K$ sein. Fallen die beiden Tangenten zusammen, so müssen sie diesen Kegelschnitt berühren. Der Pol $y$ und der Rückkehrpunkt $x$ seiner Polare müssen dann also Punkte von $S$ sein, deren Polare in eine Doppelgerade degenerirt. Da aber $x$ auf dieser Geraden liegen muss, so kann die Rückkehrtangente nur eine solche Seite eines Fünfseits sein, mit welcher eine andere zusammenfällt. $y$ ist dann einer der zu dieser Seite gehörigen Pole. Es folgt also hieraus: In einem der acht $F$ ünfseite, in welchem zwei Seiten coincidiren, ist diese Seite Rückkehrtangente von drei ersten Polaren. Die Pole dieser Polaren sind die Schnittpunkte der drei übrigen Sèiten and jede dieser berührt $S$ in dem Rückkehrpunkte der Polare des ihr gegenüberliegenden Pols. Es gibt also, wie bekannt, 24 Polaren, welche Rückkehrpunkte haben. Die Rückkehrpunkte selbst aber sind die Schnittpunkte von $S$ mit der Hesse'schen Curre, wie Herr Clebsch gezeigt hat. Diese beiden Curven schneiden sich also in 24 Punkten, welche je zu dreien auf acht Geraden liegen. Die Gleichnng $24^{\text {ten }}$ Grades, welche diese Schnittpunkte liefert, wird sich also mit Hülfe einer Gleichung achten Grades und Gleichungen dritten Grades lösen lassen.

Die acht Geraden bilden eine Curve achter Ordnutng, welehe mit einer zweiten Curve achter Ordnung, die besteht ans der Hesse'schen Curve und dem Kegelschnitt $K, 64$ Punkte gemein hat, von welchen 32 auf der Curve vierter Ordnung $S$ liegen. Nach einem bekannten Satze liegen also die übrigen 32 auf einer zweiten Carve vierter Ordnung $S_{1}$. Da aber jede der acht Geraden den Kegelschnițt $K$ in zwei 
unendlich nahen Punkten schneidet, von welchen einer nur auf $S$ liegt, so wird der andere auf $S_{1}$ liegen müssen, d. h. $S$ und $S_{1}$ sehneiden sich in 16 Punkten, von welchen 8 anf dem Kegelsehnitt $K$ liegen. Die übrigen 8 liegen dann auf einem zweiten Kegelschnitte. Zwischen den 5 Covarianten: der Hesse'schen Determinante $\Delta$, der Gleichung $K^{\prime}=0$ des Kegelschnittes in Punktcoordinaten, dem Product der acht Geraden, welches wir $P$ nennen wollen, und den beiden $S$ and $S_{1}$ findet also eine Gleichung statt von der Form:

$$
S, S_{1}+\lambda P=\mu \Delta . K^{\prime}
$$

in welcher, wie leicht ersichtlich, $\lambda$ und $\mu$ reine Zahlenfactoren sind.

\section{§. 3.}

\section{Ableitung aller Transformationen aus einer bekannten.}

Die Ausführung der Transformation der Gleichung der Curve in eine Summe ron Biquadraten erfordert, wie das Obige zeigt, die Lösung einer Gleichung vierten Grades und eines Systems linearer Gleichungen. Wenn aber eine Transformation bekannt ist, so braucht man, um die übrigen zu finden, nicht mehr auf die Gleichung der Curve $\$$ zu recur. riren, sondern man kann eine Gleichung fünften Grades mit einer willkürlicken Grösse aufstellen, welche alle anderen Transformationen liefert. Man kann bekanntlich die Gleichung einer Tangente des Kegelschnittes $K$ darstellen in der Form $a+b \lambda+c \lambda^{2}=0$, wo $\lambda$ ein Parameter ist. Bezeichnen wir die Parameter, welche den fünf Seiten eines Fünfseits zugehören, mit $\lambda_{1} \ldots \lambda_{5}$, mit $A_{i}=0$ die Gleichung der Seite, deren Parameter $\lambda_{i}$ ist, und setzen

so wird dureh

$$
\left(\lambda-\lambda_{1}\right)\left(\lambda-\lambda_{2}\right) \ldots\left(\lambda-\lambda_{5}\right)=f(\lambda)
$$

$$
B_{k}^{2}=\sum_{i} \frac{A_{i}^{2}}{f^{\prime}\left(\lambda_{i}\right)\left(l_{k}-\lambda_{i}\right)}
$$

das Quadrat der Gleichung der zum Parameter $l_{k}$ gehörigen Tangente dargestellt. Schreiben wir die fünf Gleichungen an, welche zu den noch zu suchenden Parametern $l_{1} \ldots l_{\text {s }}$ gehören, und lösen die Gleichungen auf (vergl. Baltzer, Determ., p. 88), so folgt:

wo

$$
A_{i}^{2}=-g\left(\lambda_{i}\right) \sum_{k} \frac{f\left(l_{k}\right)}{g^{\prime}\left(l_{k}\right)} \cdot \frac{B_{k}^{2}}{l_{k}-\lambda_{i}},
$$

gesetzt ist.

$$
g(l)=\left(l-l_{1}\right)\left(l-l_{2}\right) \ldots\left(l-l_{5}\right)
$$

Ist non die Gleichung unserer Curve vierter Ordnung in Bezug auf das Fünfseit der $A$.

$$
u=\underset{-i}{\sum} Q_{i} A_{i}^{4}=0
$$


so wird sie ausgedrückt in den $B$

$$
u=\sum_{i} \sum_{k} \sum_{h} \rho_{i} g\left(\lambda_{i}\right)^{2} \frac{f\left(l_{k}\right) f\left(l_{h}\right)}{g^{\prime}\left(l_{k}\right) g^{\prime}\left(l_{h}\right)} \cdot \frac{B_{h}^{2} \cdot B_{k}^{2}}{l_{k}-\lambda_{i} \cdot l_{k}-\lambda_{i}} .
$$

Sollen nun die $B$ wieder ein Fünfseit darstellen, so müssen die Producte $B_{h}^{2} B_{k}^{2}$ fortfallen und also die Gleichungen bestehen:

$$
\sum_{i} \varrho_{i} g\left(\lambda_{i}\right)^{2} \frac{1}{l_{h}-\lambda_{i} \cdot l_{k}-\lambda_{i}}=0, h \text { nicht gleich } k \text {. }
$$

Diese 10 Gleichungen können aber zusammen bestehen, denn sie entstehen durch Subtraction je zweier der fünf Gleichungen:

$$
\sum_{i} \varrho_{i} g\left(\lambda_{i}\right)^{2} \frac{1}{l_{k}-\lambda_{i}}=\mu \quad, k=1 \ldots 5,
$$

wo $\mu$ willkürlich ist. Bestimmt man aus diesen fünf Gleichungen $\rho_{i} g\left(\lambda_{i}\right)$, so ergibt sich

$$
\rho_{i} g\left(\lambda_{i}\right)=-\mu \frac{1}{f^{\prime}\left(\lambda_{i}\right)} \sum_{k} \frac{f\left(l_{k}\right)}{g^{\prime}\left(l_{k}\right)} \cdot \frac{1}{l_{k}-\lambda_{i}} .
$$

Die im zweiten Gliede auftretende Summe ist aber nach einem bekannten Satze der Partialbruchzerlegung $=1$ und also

$$
\rho_{i} g\left(\lambda_{i}\right)=-\frac{\mu}{f^{\prime}\left(\lambda_{i}\right)} .
$$

Fuht man diesen Werth in die abige Gleichung ein, so ergibt sich endlich in

$$
\frac{1}{\mu}=\sum_{i} \frac{1}{e_{i} f^{\prime}\left(\lambda_{i}\right)^{2}\left(l-\lambda_{i}\right)}
$$

eine Gleichung, die durch $l_{k}$ erfüllt sein muss und also für jeden Werth von $\mu$ die fünf Parameter der Seiten eines Fünfseits liefert, welches die gewünschte Eigenschaft besitzt. Da die Discriminante einer Gleichung fünften Grades vom achten Grade in den Coëfficienten ist, so ergibt sich anch hier, dass 8 Fünfseite existiren, in welchen zwei Seiten zusammenfallen.

\section{ร. 4.}

\section{Untersuchung der Covariante $S$.}

Bilden wir nun mit Zugrundelegung der Form (9) die Gleichung der Curve $S$. Wenn wir für die Coëfficienten einer ternären biquadratischen Form symbolisch Potenzen und Producte von Grössen $a, b, c, d$ einführen, so wird nach Herrn Aronhold der symbolische Ausdruck von $S$ gegeben durch

$$
6 S=a b c a(a b c)(a b d)(a c d)(b c d),
$$

wo $a=a_{1} x_{1}+a_{2} x_{2}+a_{3} x_{3}$ und $(a b c)=\Sigma \pm a_{1} b_{2} c_{3}$ gesetzt ist. Schreiben wir; wie im vorigen $\S ., u$ in der Form 


$$
u=\sum_{i} \varrho_{i} A_{i}^{4},
$$

and bezeichnen mit ( $i h /$ ) die Determinante der drei Formen $A_{i}, A_{k}, A_{k}$, so findet man, abgesehen ron einem Zahlenfactor:

$$
\begin{aligned}
& S=\varrho_{1} \varrho_{2} \varrho_{3} \varrho_{4}(123)(124)(134)(234) A_{1} A_{2} A_{3} A_{4} \\
& +\quad \varrho_{1} \varrho_{2} \varrho_{3} \varrho_{5}(123)(125)(135)(235) A_{1} A_{2} A_{3} A_{5} \\
& +\quad \varrho_{1} \varrho_{2} \varrho_{4} \varrho_{5}(124)(125)(145)(245) A_{1} A_{2} A_{1} A_{5} \\
& +\varrho_{1} \varrho_{3} \varrho_{4} \varrho_{5}(134)(135)(145)(345) A_{1} A_{3} A_{4} A_{5} \\
& +\quad \varrho_{2} \varrho_{3} \varrho_{4} \varrho_{5}(234)(235)(245)(345) A_{2} A_{3} A_{1} A_{5}
\end{aligned}
$$

wofür wir abkürzend schreiben wollen:

$$
S=k_{5} A_{1} A_{2} \cdot A_{3} A_{1}+k_{1} A_{1} A_{2} A_{3} A_{5}+\ldots
$$

Die Curve $S$ geht also, wie dies sein muss, durch die Ecken des Fünfseits der $A$. Die Gleichung der Tangente an $S$ im Schnittpunkte der Seiten $A_{1}=0$ und $A_{2}=0$ findet sich

$$
\frac{A_{1}}{k_{1}}+\frac{A_{2}}{k_{2}}=0 \text {. }
$$

Stellt man ebenso die Gleichungen der Tangenten auf für die beiden Ecken $A_{1}=0 \quad A_{3}=0$, und $A_{2}=0 \quad A_{3}=0$, so erkennt man leicht, dass diese drei Tangenten die gegenüberliegenden Seiten in drei Punkten einer geraden Linie schneiden. Man kann folglich einen Kegelschnitt beschreiben, der die Curve $S$ in den Ecken eines Dreiseits des Fünfseits berührt. Die Gleichung dieses Kegelschnittes wird

$$
H=k_{1} A_{2} A_{3}+k_{2} A_{3} A_{1}+k_{3} A_{1} A_{2}=0,
$$

während $S$ die Form annimmt

$$
S=A_{1} A_{2} A_{3}\left(k_{4} A_{5}+k_{5} A_{4}\right)+A_{4} A_{5} \cdot H .
$$

Diese Gleichung zeigt, dass der obige Kegelschnitt $S$ noch in zwei weiteren Punkten trifft, deren Verbindungsliniedureh den Schnittpunkt der beiden anderen Seiten des Fünfseits geht nud dort $S$ berührt. Diese Ableitung verliert ihre Gültigkeit, wenn zwei Seiten des Fünfseits zusammenfallen. Die Betrachtung der Figur zeigt dann aber sofort, dass drei der Kegelschnitte, welche den vier dann existirenden Dreiseiten entsprechen, in zwei Linien zerfallen. Dass man um das vierte Dreiseit, dessen Seiten in den Schnittpunkten der zusammenfallenden Seiten $S$ berühren, einen Kegelsehnitt legen kam, von dem der obige Satz gilt, zeigt sich mit Hiilfe des bekannten Theorems: Wenn man in den Schuittpunkten einer Carve $n^{\text {ter }}$ Ordnung bud einer Geraden die Tangenten an die Curve legt, so schneiden diese die Curve noch in $n(x-2)$ weiteren Punkten, welche auf einer Curve $(n-2)^{\text {ter }}$ Ordnung liegen.

Wir haben num oben gesehen, dass zu einem Punkte von $S$ ein einziges Fünfseit gehört. In diesem Fünfseit ist ein Dreiseit dadurch ansgezeichnet, dass seine Seiten nicht durch jenen Punkt gehen. Der 
obige Satz zeigt dann, dass der Kegelschnitt, weleher $S$ in den Ecken des Dreiseits berührt, noch in zwei Punkten schneidet, die auf der Tangente des gegebenen Punktes liegen. Wir wollen das Dreiseit und den Kegelschnitt als zu, dem Punkte gehörig bezeichnen. Betrachtet man nun die zu zwei Punkten von $S$ gehörigen Kegelschnitte und lässt den einen Purkt stetig seine Lage verändern, so wird auch der zugehörige Kegelschnitt sich stetig ändern, und wenn der eine Punkt mit dem andern zusammenfällt, so werden die beiden Kegelschnitte auch zusammenfallen. Denn wenn dies nicht der Fall wäre, so würden zwei Kegelschnitte existiren, welche zu einem Punkte gehörten, was unmöglich ist. Die zu den verschiedenen Punkten ron $S$ gehörigen Kegelscbnitte bilden also nach dem von Herrn Hesse (Crelle s Journal Bd. 49, p. $243 \mathrm{ff}$.) aufgestellten Begriffe ein und dasselbe System.

Betrachten wir nun zwei Punkte $a$ und $b$ von $S$. Da die beiden zugehörigen Dreiseite dem Kegelschnitte $K$ umschrieben sind, so liegen ihre Ecken auf einem zweiten Kegelschnitte $G$. Dieser schueidet $S$ noch in zwei weiteren Punkten. Um deren Lage zu finden, betrachten wir die zu $a$ und $b$ gehörigen Kegelschnitte $H$ und $H^{\prime}$ zusammen als eine Curve vierter Ordnung und den doppelt gerechneten Kegelschnitt $G$ in Verbindung mit den beiden Tangenten $T$ und $T^{\prime}$, die man in $a$ resp. $b$ an $S$ legen kann, als eirie Curve sechster Ordnung. Von den Schnittpunkten dieser Curve mit der Curve vierter Ordnung $S$ liegen dann 16 auf der Curve $H H^{\prime}$. Nach einem bekannten Satze von Cayley (cf. Cremona, ebene Curven, p. 65) liegen also die übrigen anf einer Curve zweiter Ordriung. Diese acht Punkte sind aber die zwei Paare $c, d$ von unendlich nahen Punkten, welche der doppelt gerechnete Kegelschnitz $G$ ausser den Berührungspunkten von $H$ und $H^{\prime}$ noch mit $S$ gemein hat, und die zwei Paare $a, b$ von unendlich naben Punkten, in welchen $T$ und $T^{\prime \prime}$ schneiden. Es müsste also ein Kegelschnitt existiren, welcher die Curve $S$ in den willkürlich gewählten Punkten $a, b$ und in noch zwei arideren $c, d$ berührte. Dies ist aber nicht möglich, weil schon die Zahl der Berührungskegelschnitte, welche in einem Punkte berühren, eine endliche ist. Der Kegelschnitt muss also zerfallen. Aber auch der Fall von zwei Linien ist zu verwerfen, weil sonst durch einen beliebigen Punkt von $S$ eine Doppeltangente zu legen wäre. Es bleibt also nur der Fall übrig, dass der Kegelschnitt eine Doppellinie-ist. Wir haben somit den Satz: Wenn man zu zwei Punkten $a$ und $b$ ron $S$ die zugehörigen Kegelsehnitte construirt, so liegen deren Berührungspunkte anf einem Kegelsehnitte, der $S$ in zwei weiteren Punkten $c$, $d$ schneidet. $a, b, c, d$ liegen dann a uf einer geraden Linie. Es folgt hieraus hoch, dass, wenn man in der Construction ausgegangen 
wäre von den beiden Punkten $c, a$, man die Punkte $a, b$ erhalten hätte, and Wenn man durch zwei Punkte $a, b$ von $S$, eine gerade Linie legt, welche $S$ noch in den beiden Punkten $e$, $d$ trifft, so schneidet der Kegelschnitt $G$, welchen man durch $c, d$ and die Ecken des zu a gehörigen Dreiseits beschreiben kann, $S$ noch in drei Punkten, welchedie Ecken des zu $b$ gehörenden Dreiseits sind.

Die oben benutzte Ueberlegung verliert ihre Gültigkeit, wenn a and $b$ Berührungspunkte einer und derselben Doppeltangente sind. Dann berührt aber der zu $a$ gehörige Kegelschnitt in $b$ und umgekehrt, und beide Kegelschnitte sind also Berührungskegelschnitte, die in aller Punkten berïhren, wo sie $S$ treffen. Da sie aber in ihrer früheren Bedeutung zum gleichen System gehören, müssen sie auch demselben System von Berührungskegelschnitten angehören. Nach einem Satze von Herm Hesse (Crelle's Journal Bd. 49, p. 262) liegen also die Berührungspunkte wieder auf einem Kegelschnitte. Es tritt daher zu dem obigen Satze noch die Ergänzung hinzu: Sind die beiden Punkte $a, b$ Berührungspunkte einer Doppeltangente, so geht derKegelschnitt, den man um die Ecken der zugehörigen Dreiecke legen kann, durch die nämlichen beiden Punkte $a, b$ hindurch.

Da die Gleichung von $S$ auch geschrieben werden kann:

$$
S=A_{5} \cdot H_{5}+k_{5} A_{1} A_{2} A_{3} A_{1}=0,
$$

wo $\boldsymbol{H}_{5}$ die Gleichung:

$$
k_{\mathrm{i}} A_{2} A_{3} A_{4}+k_{2} A_{1} A_{3} A_{4}+k_{3} A_{1} A_{2} A_{4}+k_{4} A_{1} A_{2} A_{3}=0
$$

einer Curve dritter Ordnung bezeichnet, so sieht man, dass $S$ von einer Curve dritter Ordnung berührt wird in den Ecken eines Vierseits. Da das Vierseit durch eine Tangente an $K$ eindentig bestimmt ist, so folgt, dass alle Berührungscurven dritter Ordnung derart einem Systeme angehören. Die hier auftretende, Curve $H_{5}$ hat noch eine besondere Bedeutung. In der allgemeinen Theorie tritt eine Zwischenform auf, deren Symbol ist:

$$
S_{\iota c}=(u a b)(u a c)(u b c)(a b c) a b c \text {; }
$$

führen wir hier unsern Aasdruck (9) für $u$ ein, so erhalten wir, abgesehen ron einem numerisehen Factor:

$$
S_{\psi}=\sum_{x \lambda \mu} \varrho_{\alpha} \varrho_{\lambda} \varrho_{\mu}(\varkappa \lambda \mu)(u x \lambda)(u x \mu)(u \lambda \mu) A_{*} A_{\lambda} A_{\mu},
$$

wo die Summe ausgedehnt ist über die Combinationen ohne Wiederholungen der Zahlen 1,2, 3, 4,5 zu je dreien, und wo $(u x \lambda)$ die Determinante von $A_{x}, A_{2}$ und der Form $u_{1} x_{1}+u_{2} x_{2}+u_{3} x_{3}$ bezeichnet. Setzen wir für $u_{1} u_{2} u_{3}$ die Coordinaten der Linie $A_{3}$, so geht, wie man sieht, $S_{w}$ über in $H_{3}$. Da $A_{5}$ eine beliebige Tangente des Kegel- 
schnittes $K$ darstellt, so folgt mit Rücksicht auf die a. a. $O$. gegebene Bedeutung von $S_{u}$ der Satz: Die Punkte, deren erste Polaren von einer gegebenen Tangente des Kegelschnittes $K$ in solchen Punkten geschnitten werden, dass die in den Schnittpunkten an die Polare gezogenen Tangenten sich in einem Punkteschneiden, liegen auf ejner Curve dritter Ordnung, welche $S$ in den Ecken des zur gegebenen Tangente gehörigen Vierseits berïhrt.

Bezeichnen wir mit $\varrho$ einen beliebigen linearen Ausdruck der Coordinaten, so stellt die Gleichung

$$
\varrho S+A_{1} A_{2} A_{3} A_{4} A_{5}=0
$$

ein ganzes Netz von Curven fünfter Ordnung dar, dessen Curven alle in den Eckpunkten eines Fünfseits $S$ berühren. Die Eckpunkte zweier Fünfseite gehören zum gleichen System von Berührungspunkten. Denn da die Eckpunkte durch einen unter ihnen eindeutig bestimmt sind, so müssen sie alle zusammenfallen, wenn man einen Punkt des einen Fünfseits durch stetige Aenderung mit einem des anderen zur Deckung bringt.

Zum Beschlusse dieser Betrachtungen will ich noch den folgenden Satz anführen:

Wenn eine gegebene Curve vierter Ordnung die Eigenschaft hat, dass man ein vollständiges Fünfseit beschreiben kann, dessen Ecken alle auf ihr liegen, so kann man sie betrachten als Covariantencurve $S$ zu einer anderen Curve vierter Ordnung, von der in $\$$. 1. charakterisirten Art. In der That kann dann, wenn mit $A_{1} \ldots A_{5}$ die Gleichungen der Seiten bezeichnet werden, die Gleichung der Curve stets in die Form gesetzt werden:

$$
\begin{aligned}
0=\varrho_{1}^{\prime} A_{2} A_{3} A_{4} A_{5}+\varrho_{2}^{\prime} A_{1} A_{3} A_{4} A_{5} & +\varrho_{3}^{\prime} A_{1} A_{2} A_{4} A_{5}+\varrho_{4}^{\prime} A_{1} A_{2} A_{3} A_{5} \\
& +\varrho_{5}^{\prime} A_{1} A_{2} A_{3} A_{4},
\end{aligned}
$$

wo die $\varrho_{1}^{\prime} \ldots \varrho_{5}^{\prime}$ bestimmte Coëfficienten sind. Die Vergleichung mit der Form $\left(11^{*}\right)$ der Gleichung von $S$ zeigt aber dann sofort, dass dieses die Covariante $S$ ist zu der Form:

$$
\frac{k_{1}}{\varrho_{1}^{\prime}} A_{1}^{4}+\frac{k_{2}}{\varrho_{2}^{\prime}} A_{2}^{4}+\frac{k_{3}}{\varrho_{3}^{\prime}} A_{3}^{4}+\frac{k_{4}}{\varrho_{4}^{\prime}} A_{4}^{4}+\frac{k_{5}}{\varrho_{5}^{\prime}} A_{5}^{4} .
$$

Es gelten somit alle hier bewiesenen Eigenschaften ron $S$ allgemein für jede der Curven der in letzten Satze bezeichneten Art. Einen Satz hebe ich noch hervor, der aus dem Vorigen sich sofort ergibt: Wenn man einer Curve vierter Ordnung ein Fünfseit einschreibenkann, so kann man ihr unendlich viele einschreiben, deren Seiten alle einen Kegelschnitt berühren.

Nach einer blossen Abzählung könnte es scheinen, dass es stets möglich wäre, einer Curve vierter Ordnung ein solches Fünfseit ein- 
zuschreiben. Der vorletzte Satz zeigt, dass dies nicht richtig ist, denn eine allgemeine Curve vierter Ordnung müsste dann von einer anderen mit nur 13 Constanten abhängen, was unmöglich ist.

\section{§. 5.}

\section{Ausnahmefalle.}

Die Resultate, welche in den vorhergehenden \$§. erlangt sind, stützen sich auf die Voraussetzung, dass der Kegelschnitt $K$ nicht zerfällt. Wir wollen nụn noch in Kürze die Aenderungen angeben, welche eintreten, wenn diese Voraussetzung nicht mehr gültig ist.

Der Kegelschnitt $K$ möge zunächst bestehen aus zwei Punkten $a$ und $b$. Damit dies der Fall sei, muss die Determinante der $p_{i k}$ verschwinden. Die Coordinaten dieser beiden Punkte genügen dann den Gleichungen:

$$
\sum_{i, k} a_{i} b_{k} u_{i k m_{m}}=0, l, m=1,2,3,
$$

welche sofort aussagen, dass die erste Polare ron $a$ im Punkte $b$, und die von $b$ im Punkte $a$ einen dreifachen Punkt besitzt, d. h. dass diese Polaren in drei gerade Linien zerfallen. Beziehen wir nun einen Punkt durch seine Coordinaten $x y z$ auf ein Dreieck, dessen Ecken $x=0 y=0$ und $x=0 z=0$ in den Punkten $a$ und $b$ resp. liegen, während die dritte Ecke einer der Punkte ist, in welchen sich die Polare von $a$ und die von $b$ schneiden, so ist die Gleichung der ersten Polare von $a u_{3}=0$, wo nun $u_{3}$ eine binäre Form der Variabeln $x$ und $z$ ist, und ebenso ist die Polare von $b \quad u_{2}=0$ und $u_{2}$ enthält nur die Variabeln $x$ und $y$. Wenn wir also die gewöhnliche Bezeichnungsweise der dritten Differentialquotienten von $u$ anwenden, so ist

$$
u_{223}=u_{332}=u_{123}=0,
$$

und die Gleichung der Curve kann in die einfachere Form gesetzt werden: (12) $a x^{4}+b y^{4}+c z^{4}+4 b^{\prime} x y^{3}+4 c^{\prime} x z^{3}+6 b^{\prime \prime} x^{2} y^{2}+6 c^{\prime \prime} x^{2} z^{2}=0$. Berechnet man nun den Ausdruck von $S$ nach der von Herrn Aronhold gegebenen Darstellung, so folgt, mit Berücksichtignng der obigen Gleichungen and mit Fortlassung eines Zahlenfactors:

$$
S=\left(u_{122}^{2}-u_{112} \cdot u_{222}\right)\left(u_{133}^{2}-u_{113} \cdot u_{333}\right) \text {. }
$$

Der erste Factor $u^{2}{ }_{122}-u_{112} \cdot u_{222}$ ist die Hesse'sche Determinante der binären Form $u_{2}$, der zweite dieselbe Covariante von $u_{3}$. Wir seben also, dass hier die Covariante $S$ in $z w e i$ Linienpare zexfällt, die ihre Scheitel in $a$ and $b$ haben und die cyclischprojectivischen Linien sind zu den Linien, welche die ersten Polaxen von $b$ und $a$ bilden. Wo der Ausdruck cyclisch-projec- 
tivisch nach Herrı Clebsch (Crelle's Journal Bd. 68, p. 167) gebraucht ist, um die bekannte Beziehung anzudeuten, in welcher die Linien der Hesse'schen Determinante zu den Linien der Form selbst stehen. Es ist nun aus der Theorie der binären Formen dritten Grades bekannt, dass durch Einführung der Factoren der Hesse'schen Covariante als neuer Variabelen die Form sich darstellt als Summe zweier Cuben. Setzt man also $u^{2}{ }_{112}-u_{122} u_{222}=Y_{1} Y_{2}$, wo $Y_{1} Y_{2}$ lineare Ausdrücke in $x$ und $y$, so wird

und ähnlich geht $u_{3}$ über in

$$
u_{2}=\alpha Y_{1}^{3}+\beta Y_{2}^{3}
$$

$$
u_{3}=\alpha^{\prime} Z_{1}^{3}+\beta^{\prime} Z_{2}^{3} \text {. }
$$

wenn wir $u_{113}^{2}-u_{133} u_{333}=Z_{1} Z_{2}$ setzen. Durch Integration ergibt sich hieraus für $u$ die Form:

$$
u=A^{\prime} x^{4}+B_{1} Y_{1}^{4}+B_{2} Y_{2}^{4}+C_{1} Z_{1}^{4}+C_{2} Z_{2}^{4},
$$

so dass sich auch jetzt noch $u$ als Summe von fünf Biquadraten darstellen lässt. Aus dieser einen Darstellung lassen sich noch unendlich viol andere ableiten. Man kann nämlich entweder die binäre Form

$$
A^{\prime} x^{4}+B_{1} Y_{1}^{4}+B_{2} Y_{2}^{4}
$$

oder die Form

$$
A^{\prime} x^{4}+C_{1} Z_{1}^{4}+C_{2} Z_{2}^{4}
$$

wieder auf unendlich viele Arten als Summe von drei Biquadraten darstellen, und erhält dann neue Ausdrücke für $u$. Die in einer solchen Darstellung angewandten Linien scheiden sich in zwei Grupperr von zwei und drei Linien. Das Linienpaar der ersten Groppe hat seinen Scheitel in einem der beiden Punkte $a, b$ und ist identisch mit dem ron hier ausgehenden Linienpaar von $S$. Die drei Linier der zweiten Gruppe gehen dann dureh den zweiten Punkt.

Die in (14) gegebene Darstellung wird unmöglich, wenn die Polare von $b$ z. B. aus drei Linien besteht, von welchen zwei zusammenfallen. Dann kann man $u_{2}$ nicht mehr auf die oben angenommene Form bringen, sondern muss setzen.

$$
u_{2}=Y_{1}^{2} Y_{2} \text {. }
$$

Hieraus folgt für den von $y$ abhängigen Theil von $u$ die Form:

$$
B Y_{1}^{3} Y_{2}+B^{\prime} Y_{1}^{4} \text {. }
$$

Dagegen lässt sich $u$ sogar als Summe von vier Biquadraten darstellen, wenn die Polare eines der Punkte $a, b$, des letzteren z. B., aus drei znsammenfallenden Linien besteht. Denn da $u_{2}$ dann in die Form gebracht werden kamn

so hat den Ausdruck

$$
u_{2}=Y^{3} \text {, }
$$

$$
u=A^{\prime} x^{4}+B Y^{4}+C_{1} Z_{1}^{4}+C_{2} Z_{2}^{4},
$$


der sich durch den oben schon angewandten Process noch auf unendlich viele andere Formen bringen lässt.

Eine Daristellung der Gleichung der Curve als Summe von vier vierten Potenzen tritt auch dann ein, wenn in dem Ausdruck (14) die Constante $A^{\prime}$ verschwindet. Dieser Fall unterscheidet sich aber von dem vorhergehenden dadurch, dass die Gleichung $u=0$ sich nur anf eine Weise in diese Form bringen lässt. Denn eine zweite köunte nur daraus hervorgehen, dass man die Summe zweier der vorkommenden vierten Potenzen noch in anderer Weise in der gleichen Form ausdrückte. Dies ist aber unmöglich; denn wenn eine binäre form vierten Grades als Summe von zwei Biquadraten dargestellt werden kann, so ist dies nur auf eine Weise möglich. Was nun die Bedingungen dieses Falles betrifft, so erkennt man leicht, dass, wenn man die Schnittpunkte der zwei Linienpaare, welche $S$ bilden, bezeichnet mit $a^{\prime} b^{\prime}, a^{\prime \prime} b^{\prime \prime}$, die Polaren des Punktenpaares $a^{\prime} b^{\prime}$ und die des Punktenpaares $a^{\prime \prime} b^{\prime \prime}$ unbestimmt werden; und umgekehrt, wenn dies der Fall ist, so zeigt die Gleichung (14), dass $A^{\prime}=0$ ist. Es findet daher nicht nur die Gleichung statt

sondern auch die beiden andern

$$
\sum a_{i} b_{k} u_{i k i m}=0,
$$

$$
\begin{aligned}
& \Sigma a_{i}^{\prime} b_{k}^{\prime} u_{i k l m}=0, \\
& \Sigma a_{i}^{\prime \prime} b_{k}^{\prime \prime} u_{i k l m}=0 .
\end{aligned}
$$

Wenn andererseits diese Gleichungen bestehen, so zeigt die Untersuchung am Beginı dieses $\$$., dass $a, b, a^{\prime}, b^{\prime}, a^{\prime \prime}, b^{\prime \prime}$ Doppelpunkte von $S$ sein müssen, d. h. dass in der That vier dieser Punkte die Schnitipunkte zweier Linienpaare sind, welche von den beiden anderen ausgehen. Die Gleichungen oben aber sind nur möglich, wenn die

$$
\Sigma p_{i k} u_{2 k i m}=0
$$

Auflösungen Hefern von der Form

$$
p_{i k}=p_{i k}^{\prime}+\lambda p_{i k}^{\prime \prime}
$$

wo $\lambda$ beliebig ist. Dann kann man in der That auf drei Arten $\lambda$ so bestimmen, dass der Kegelschnitt $K$ ein Punktenpaar wird. Auflösungen von dieser Art setzen aber bekanntlich voraus, dass ausser der Determinante der Gleichungen anch noch sämmtliche erste Unterdeterminanten verschwinden, während mindestens eine zweite Unterdeterminante nicht gleich Null sein darf: (Vergl. Baltzer, Determ. p. 62.)

Olne mich bei den anderen leicht zu übersehenden Aenderungen aufzuhalten; die noch eintreten können, will ich noch den Fall betrachten, dass der Kegelschmitt $K$ zerfällt in einen doppelt zu rechnenden Punkt a. Dann bestehen die Gleichungen

$$
\Sigma a_{i} a_{k} u_{i k i k z}=0,
$$


welche aussagen, dass die Curve einen dreifachen Punkt hat in $a$. Dass auch der umgekehrte Schluss berechtigt ist, ist klar; es ergibt sich hiexaus der Satz: Wenn eine Curve vierter Ordnung einen dreifachen Punkt haben soll, so muss exstens die" Determinante $A$ verschwinden. Dann sind die Unterdeterminanten Producte je zweier ron sechs Grössen $p_{i k}$. Es müssen dann zweitens sämmtliche Determinanten zweiten Grades verschwinden, welche man aus diesen $p_{i k}$ bilden kann.

Dieser Satz ist ein specieller Fall eines allgemeinen, welcher für Formen geraden Grades von beliebig vielen $(n)$ Variabeln gilt. Soll eine solche Form $2 p^{\text {ten }}$ Grades eine $(p+1)$ fache Lösung besitzen, so muss ein System ron $(n, p)$ Formen $p^{\text {ten }}$ Grades gleichneitig annullirt werden, wo $(n, p)=\frac{n \cdot n+1 \ldots n+p-1}{1.2 \ldots p}$. Diese Zahl ist aber die Anzahl der Glieder einer Form $p^{\text {ten }}$ Grades. Aus den aufgestellten Gleichungen kann man also die Variabeln eliminiren, und erhält als erste Bedingung, dass eine Determinante verschwinden muss, welche $\operatorname{der} A$, die wir hier betrachteten, ganz analog gebildet ist. Die ersten Unterdeterminanten dieser Determinante sind dann Producte je zweier von $(n, p)$ Grössen, die man als die Coëfficienten einer zugebörigen Form $p^{\text {tes }}$ Grades auffassen kann. Wenn diese zugehörige Form eine $p^{\text {te }}$ Potenz eines linearen Ausdrucks ist, so geben die Coëfficienten dieses Ausdrucks die Werthe der Variabeln, für welche die $(p+1)$ fache lösung stattfindet. Die gesuchten Bedingungen lassen sich also aufstellen, wenn man die Bedingungen angeben kann, dass eine Form $p^{\text {ten }}$ Grades die $p^{\text {te }}$ Potenz eines linearen Ausdrucks ist.

Heidelberg, den 27. Juni 1868. 